\title{
Rainbow degree-jump coloring of graphs
}

\author{
Mphako-Banda E.G. ${ }^{1}$, Kok J. ${ }^{2}$, Naduvath S. ${ }^{2}$
}

\begin{abstract}
In this paper, we introduce a new notion called the rainbow degree-jump coloring of a graph. For a vertex $v \in V(G)$, let the degree-jump closed neighbourhood of this vertex be defined as $N_{d e g}[v]=\{u: d(v, u) \leq d(v)\}$. A proper coloring of a graph $G$ is said to be a rainbow degree-jump coloring of $G$ if for all $v$ in $V(G), c\left(N_{d e g}[v]\right)$ contains at least one of each color class. We determine a necessary and sufficient condition for a graph $G$ to permit a rainbow degree-jump coloring. We also determine the rainbow degree-jump chromatic number, denoted by $\chi_{r d j}(G)$, for certain classes of cycle related graphs.

Key words and phrases: rainbow degree-jump coloring, rainbow degree-jump chromatic number, blind vertex, Mphako graph, Moore bound.
\end{abstract}

\footnotetext{
${ }^{1}$ School of Mathematical Sciences, University of Witswatersrand, Johannesburg, South Africa

2 Department of Mathematics, CHRIST (Deemed to be University), Bangalore, Karnataka, India

E-mail: eunice.mphako-banda@wits.ac.za (Mphako-Banda E.G.), johan.kok@christuniversity.in (KokJ.), sudev.nk@christuniversity.in (Naduvath S.)
}

\section{Introduction}

For general notations and concepts in graphs and digraphs see [1,3,9]. Unless mentioned otherwise all graphs $G$ are simple, connected and finite.

For a set of distinct colors $\mathcal{C}=\left\{c_{1}, c_{2}, c_{3}, \ldots, c_{\ell}\right\}$, a vertex coloring of a graph $G$ is an assignment $\varphi: V(G) \mapsto \mathcal{C}$. A vertex coloring is said to be a proper vertex coloring of a graph $G$ if no two distinct adjacent vertices have the same color. The cardinality of a minimum set of solid colors in a proper vertex coloring of $G$ is called the chromatic number of $G$ and is denoted $\chi(G)$. A coloring with exactly $\chi(G)$ colors may be called a $\chi$-coloring or a chromatic coloring of $G$. By the term $c(G)$, we mean the set $c(V(G))$ and hence we have $c(G)=\mathcal{C}$ and $|c(G)|=|\mathcal{C}|$. For a set of vertices $X \subseteq V(G)$, the coloring of the induced subgraph $\langle X\rangle$ is denoted by $c(\langle X\rangle)$ and this coloring will be permitted by $\varphi: V(G) \mapsto \mathcal{C}$.

Index labeling the elements of a graph such as the vertices say, $v_{1}, v_{2}, v_{3}, \ldots, v_{n}$ or written as $v_{i} ; 1 \leq i \leq n$ or as $v_{i} ; i=1,2,3, \ldots, n$, is called a minimum parameter indexing of $G$. Similarly, a minimum parameter coloring of a graph $G$ is a proper coloring of $G$ which consists of the colors $c_{i}, 1 \leq i \leq \ell$, where $\ell=\chi(G)$. The set of vertices of $G$ having the color $c_{i}$ is said to be the color class of $c_{i}$ in $G$ and is denoted by $\mathcal{C}_{i}$. Unless stated otherwise, we consider minimum parameter coloring throughout this paper.

Recall that the neighbourhood (or open neighbourhood) of a vertex $v \in V(G)$, denoted by $N(v)$, is the set $N(v)=\{u: v u \in E(G), u \neq v\}$. Similarly, the closed neighbourhood of $v$, denoted by $N[v]$, is the set $N[v]=N(v) \cup\{v\}$. A rainbow neighbourhood in a graph $G$ is a closed neighbourhood of a vertex $v$ in $G$ for which $c(N[v])$ contains at least one color from each color

$\mathrm{y} \Delta \mathrm{K} 519.1,519.17,519.174,519.178$

2010 Mathematics Subject Classification:05C15, 05C38, 05C75, 05 C85. 
class with respect to the chromatic coloring under consideration. For some initial works on the rainbow neighbourhoods of graphs, we refer to [4-7].

\section{Rainbow degree-jump coloring}

The concept of a rainbow neighbourhoods in a graph is specialised to what is called the rainbow degree-jump coloring of a graph. Let the degree-jump closed neighbourhood of $v$, denoted by $N_{d e g}[v]$, be defined as $N_{d e g}[v]=\{u: d(v, u) \leq d(v)\}$. Then the notion of degree-jump coloring of a graph $G$ is defined as follows.

Definition 1. A rainbow degree-jump coloring of a graph $G$ is a proper coloring of $G$ such that the degree-jump closed neighbourhood $c\left(N_{\text {deg }}[v]\right)$ of every vertex $v$ in $V(G)$ contains at least one of each color in the coloring set.

Definition 2. The maximum number of colors in a proper coloring of a graph $G$ which results in every vertex to yield a rainbow degree-jump coloring is called the rainbow degree-jump chromatic number of $G$. This new invariant is denoted by $\chi_{r d j}(G)$.

Clearly, the following are immediate observations on the rainbow degree-jump chromatic number of a graph $G$.

(i) $\chi_{r d j}(G) \geq \chi(G)$;

(ii) $\chi_{r d j}(G)=\chi(G)$ for bipartite graphs with some pendant vertices and complete graphs;

(ii) if $G$ has a pendant vertex $v$ then $N_{d e g}[v]=N[v]$.

For these graphs, the restriction on any rainbow degree-jump coloring of $G$ is $d(v, u) \leq 1$. Hence, $\chi_{r d j}(G)=\chi(G)$.

Not all graphs permit a rainbow degree-jump coloring. For example, any proper coloring of a complete graph $K_{n}, n \geq 3$, is an $n$-coloring. If pendant vertices are attached to obtain a thorny complete graph $G_{n}^{\star}$, then each pendant vertex $v$ has $N_{d e g}[v]=N[v]$. Hence,

$$
\left|c\left(N_{d e g}[v]\right)\right|=2<3 \leq n .
$$

Theorem 1. For two graphs $G$ and $H$ of order $n$ and $m$ respectively, we have

$$
\chi_{r d j}(G+H)=n+m,
$$

where $G+H$ is the join of $G$ and $H$.

Proof. For all vertices $v$ in $V(G)$ we have $d_{G+H}(v)=d_{G}(v)+m$, for all vertices $u$ in $V(H)$ we have $d_{G+H}(u)=d_{H}(u)+n$, and all pairs of vertices in $G+H$ are at a distance at most 2 . Also, the degree of all vertices in $G+H$ are greater than or equal to 2 and hence we have $\left|N_{\text {deg }}[v]\right|=n+m$ for all $v \in V(G+H)$. Hence, the result.

Recall that a clique of a graph $G$ is an induced complete subgraph in $G$. The clique number $\omega(G)$ is the order of the largest clique of $G$. Then, we have the following result. 
Theorem 2. If a graph $G$ permits a rainbow degree-jump coloring, then we have

$$
\min \left\{\left|c\left(N_{\text {deg }}[v]\right)\right|: d(v)=\delta(G)\right\} \geq \omega(G) .
$$

Proof. Let $\ell=\min \left\{\left|c\left(N_{d e g}[v]\right)\right|: d(v)=\delta(G)\right\}$. Note that any proper coloring of a largest induced complete graph of $G$ requires $\omega$ colors and $\chi(G) \geq \omega(G)$. Since a rainbow degreejump coloring is defined in terms of well-defined conditions set on all vertices and the vertex degrees, we have $\ell=\chi_{r d j}(G) \geq \chi(G) \geq \omega(G)$. It implies that if a graph permits a rainbow degree-jump coloring, then a corresponding proper $\ell$-coloring exists such that $\ell \geq \omega(G)$. This completes the proof.

Recall that a weakly perfect graph is a graph for which $\omega(G)=\chi(G)$. Then the following result is an immediate consequence of the above theorem.

Corollary 1. For a graph $G$, if $\min \left\{\left|c\left(N_{d e g}[v]\right)\right|: d(v)=\delta(G)\right\}=\omega(G)$, then $\chi(G)=\omega(G)$ and $G$ is weakly perfect.

Theorem 3. For a cycle graph $C_{n}, n \geq 3$, we have $3 \leq \chi_{r d j}\left(C_{n}\right) \leq 5$.

Proof. It is easy to verify that $\chi_{r d j}\left(C_{3}\right)=3, \chi_{r d j}\left(C_{4}\right)=4$ and $\chi_{r d j}\left(C_{5}\right)=5$. For $n \in \mathbb{Z}_{3}$, any cycle $C_{n}$ permits a proper 3-coloring such that for every vertex $v$ in $V\left(C_{n}\right), c\left(N_{\text {deg }}[v]\right)$ contains colors $c_{1}, c_{2}, c_{3}$. Hence, $\chi_{r d j}\left(C_{n}\right) \geq 3$. Since $\max \left|c\left(N_{\text {deg }}[v]\right)\right|=5$ for every vertex $v$ in $V\left(C_{n}\right)$, it follows that $3 \leq \chi_{r d j}\left(C_{n}\right) \leq 5$.

For the next corollary, let us partition the subset of positive integers as follows

$$
\mathbb{N}_{6}=\{n: n \geq 6\}=X_{1} \cup X_{2}, \quad \text { where } \quad X_{1}=\{a: 5 \mid a\}, \quad X_{2}=\{a: 5 \nmid a\} .
$$

Corollary 2. (i) For $n \in X_{1}, \chi_{r d j}\left(C_{n}\right)=5$;

(ii) If $n \in X_{2}$, then $3 \leq \chi_{r d j}\left(C_{n}\right) \leq 4$.

Proof. (i) Let the vertices of a cycle graph $C_{n}$ be labeled by $v_{1}, v_{2}, v_{3}, \ldots, v_{n}$ consecutively in the clockwise direction. Also, let $n=5 k, k \geq 1$. As $\left|N_{d e g}[v]\right|=5$, for every vertex $v$ in $V\left(C_{n}\right)$, we have $\chi_{r d j}\left(C_{n}\right) \leq 5$. However, the coloring defined by $c\left(v_{j}\right)=c_{i}$, where $j \equiv i(\bmod 5)$, with respect to which the color string $c_{1}, c_{2}, c_{3}, c_{4}, c_{5}$ consecutively repeated $k$ times, is a permissible rainbow degree-jump coloring. Hence, we have $\chi_{r d j}\left(C_{n}\right)=5$.

(ii) This result follows as a direct consequence of Theorem 3 and Part (i) written above.

Lemma 1. A graph $G$ having at least one pendant vertex with $\chi(G) \geq 3$ does not permit a rainbow degree-jump coloring.

Proof. A pendant vertex $v \in V(G)$ has $N_{d}[v]=N[v]$. Hence, $\left|c\left(N_{d}[v]\right)\right|=2<3$. Therefore, $G$ does not permit a rainbow degree-jump coloring.

\subsection{Rainbow neighbourhood jump-coloring of some cycle related graphs}

Recall the definitions of certain cycle related graph classes (see $[2,8])$ as given below.

(i) A wheel graph denoted by $W_{1, n}$, is the graph defined by $W_{1, n}=K_{1}+C_{n}$. The cycle $C_{n}$ of the wheel $W_{1, n}$ is called its rim. 
(ii) A double wheel graph, denoted by $D W_{1, n}$, is the graph obtained from two equal order wheel graphs by merging the central vertices to have a common central vertex.

(iii) A helm graph, denoted $H_{n}$, is the graph obtained from a wheel graph $W_{1, n}$ by attaching a pendant vertex to each vertex of its rim.

(iv) A closed helm graph, denoted by $\mathrm{CH}_{n}$, is the graph obtained from a helm graph $H_{n}$ by adding an edge between the pendant vertices such that these edges joining pendant vertices induces a cycle (external cycle).

(v) A prism graph $\Pi_{n}$ is the Cartesian product of a path of length 2 and a cycle. That is, $\Pi_{n}=C_{n} \square P_{2}$.

(vi) A web graph, denoted by $W_{n}$, is the graph obtained from a prism graph $\Pi_{n}$ by attaching a pendant vertex to every vertex of one of the two cycles in it.

(vii) A flower graph, denoted by $F_{n}$, is the graph obtained from a helm graph $H_{n}$ by joining the pendant vertices with its central vertex.

(viii) A djembe graph, denoted by $D j_{n}$, is the graph obtained from a prism $\Pi_{n}$ by joining all its vertices to a new external vertex (this vertex may be called the central vertex of $D j_{n}$ ). That is, $D j_{n}=\Pi_{n}+K_{1}$.

The following result discusses the rainbow degree-jump chromatic number of the abovementioned cycle related graph classes.

Proposition 1. (i) For a wheel graph $W_{1, n}$ we have $\chi_{r d j}\left(W_{1, n}\right)=n+1$.

(ii) For a double wheel graph $D W_{1, n}$ we have $\chi_{r d j}\left(D W_{1, n}\right)=2 n+1$.

(iii) A helm graph $H_{n}$ does not permit a rainbow degree-jump coloring.

(iv) For a closed helm graph $\mathrm{CH}_{n}$ we have $\chi_{\text {rdj }}\left(\mathrm{CH}_{n}\right)=2 n+1$.

(v) For a prism graph $\Pi_{n}$ we have $\chi_{r d j}\left(\Pi_{n}\right)=2 \cdot \chi_{r d j}\left(C_{n}\right)$.

(vi) A web graph $W_{n}$ does not permit a rainbow degree-jump coloring.

(vii) For a flower graph $F_{n}$ we have $\chi_{r d j}\left(F_{n}\right)=2 n+1$.

(viii) For a djembe graph $D j_{n}$ we have $\chi_{r d j}\left(D j_{n}\right)=2 n+1$.

Proof. (i) Let $u$ be the central vertex of the wheel graph and let the cycle vertices be $v_{i}, 1 \leq i \leq n$. Since $d\left(u, v_{i}\right)=1,1 \leq i \leq n$, and $d\left(v_{i}, v_{j}\right) \leq 2,1 \leq i, j \leq n$, and $d\left(v_{i}\right)=3, \forall i$ it follows that $N_{\text {deg }}\left[v_{i}\right]=N_{d e g}[u]=V\left(W_{1, n}\right)$. Hence, $r_{r d j}\left(W_{1, n}\right)=n+1$.

(ii) This result follows by similar reasoning to that in (i).

(iii) Because $\chi\left(W_{1, n}\right) \geq 3$ the helm graph which is a thorny wheel has pendant vertices. Hence, the result from Lemma 1.

(iv) Since $d\left(v_{i}\right)=4,1 \leq i \leq n$, and the inner cycle vertices, and $d\left(v_{i}, u\right) \leq 3, u \in V\left(C H_{n}\right)$, the result follows by similar reasoning to that in (i). 
(v) For a prism graph $\Pi_{n}, n \geq 3$, call the vertices $v_{i}$ from the one cycle, and $u_{i}$ from the other cycle, which are adjacent to $v_{i}$, a pair of prism images. Color any cycle in accordance with a permissible rainbow degree-jump coloring. If $c\left(v_{i}\right)=c_{j}$ color the corresponding prism image to be $c_{j+\chi_{r d j}\left(C_{n}\right)}$. It is easy to verify that

$$
\left\{c_{1}, c_{2}, \ldots, c_{\chi_{r d j}\left(C_{n}\right)}, c_{1+\chi_{r d j}\left(C_{n}\right)}, c_{2+\chi_{r d j}}\left(C_{n}\right), \ldots, c_{2 \cdot \chi_{r d j}\left(C_{n}\right)}\right\}
$$

is a permissible rainbow degree-jump coloring.

(vi) As a web graph $W_{n}$ is not 2-colorable and has pendant vertices it does not permit a rainbow degree-jump coloring.

(vii) As the pendant vertices of a helm graph are all joined to the central vertices, we have $\min \{d(u, v)\}=2$ for all pairs $(u, v)$. Therefore, the result is immediate.

(viii) The result follows by the same reasoning as in (vii).

It is obvious that if each graph $G_{i}, 1 \leq i \leq t$, permits a rainbow degree-jump coloring, then the disjoint union $\bigcup_{i=1}^{t} G_{i}$ permits such a coloring as well. Now, join the graphs in a connected string graph $G$ by adding any edge between $G_{i}, G_{i+1}, 1 \leq i \leq t-1$. Since all colorings are minimum parameter colorings, it is obvious that $\chi_{r d j}(G) \geq \chi_{r d j}\left(G_{i}\right)$, where $1 \leq i \leq t$. Note that if different combinations are stringed to obtain, say $G^{\prime}, G^{\prime \prime}$, then it is possible to find the inequality $\chi_{r d j}\left(G^{\prime}\right) \neq \chi_{r d j}\left(G^{\prime \prime}\right)$.

The following theorem characterises a graph which permits a rainbow degree-jump coloring.

Theorem 4. A graph $G$ permits a rainbow degree-jump coloring if and only if for $v, u \in V(G)$

$$
c\left(\left\langle N_{\text {deg }}[v]\right\rangle\right)=c\left(\left\langle N_{\text {deg }}[u]\right\rangle\right) \quad \text { or } \quad\left|c\left(N_{\text {deg }}[v]\right)\right|=\left|c\left(N_{\text {deg }}[u]\right)\right|
$$

with respect to some proper coloring of $G$.

Proof. If $G$ permits a rainbow degree-jump coloring $c(G)=\mathcal{C}$, then from Definition 1, it follows that $c\left(\left\langle N_{\text {deg }}[v]\right\rangle\right)=c\left(\left\langle N_{\text {deg }}[u]\right\rangle\right)=\mathcal{C}, v, u \in V(G)$, because sets are compared. Also, $c\left(\left\langle N_{\text {deg }}[v]\right\rangle\right)=c\left(\left\langle N_{\text {deg }}[u]\right\rangle\right) \Leftrightarrow v, u \in V(G)\left|c\left(N_{\text {deg }}[v]\right)\right|=\left|c\left(N_{\text {deg }}[u]\right)\right|, v, u \in V(G)$.

Since $c\left(\left\langle N_{\text {deg }}[v]\right\rangle\right)=c\left(\left\langle N_{\text {deg }}[u]\right\rangle\right)$ implies $\left|c\left(N_{\text {deg }}[v]\right)\right|=\left|c\left(N_{\text {deg }}[u]\right)\right|, v, u \in V(G)$, the desired proper coloring is obtained by initialising the proper coloring $c: V(G) \mapsto \mathcal{C}=$ $c\left(\left\langle N_{\text {deg }}[v]\right\rangle\right)$ and maximising on the coloring in accordance with the definition if $\mathcal{C}$ itself is not a maximum. The aforesaid is always possible. above.

If $\left|c\left(N_{\text {deg }}[v]\right)\right|=\left|c\left(N_{\text {deg }}[u]\right)\right|$ and $c\left(\left\langle N_{\text {deg }}[v]\right\rangle\right)=c\left(\left\langle N_{\text {deg }}[u]\right\rangle\right)$, then the result follows as

If $\left|c\left(N_{\text {deg }}[v]\right)\right|=\left|c\left(N_{\text {deg }}[u]\right)\right|$ and $c\left(\left\langle N_{\text {deg }}[v]\right\rangle\right) \neq c\left(\left\langle N_{\text {deg }}[u]\right\rangle\right)$, then without loss of generality, the coloring of $N_{d e g}(u)$ can be relabeled to obtain $c\left(\left\langle N_{d e g}[v]\right\rangle\right)$ and if need be the coloring of $N_{d e g}[v]$ can be rotated until we obtain $c\left(\left\langle N_{\text {deg }}[v]\right\rangle\right)=c\left(\left\langle N_{\text {deg }}[u]\right\rangle\right)$. This is possible unless the subgraph induced by $N_{d e g}(v) \cup N_{d e g}(u)$ is complete. But then we have a contradiction. Then, by mathematical induction, it follows that the procedure is possible for all vertices in $G$. Finally, by considering the proper coloring obtained as the initializing coloring and maximizing it if possible, the result follows. 


\section{Blind vertices in respect of degree-jump}

Theorem 2 suggests the notion of blind vertices in respect of degree-jump which is defined as follows.

Definition 3. For a vertex $v$ in $G$, if there exists a vertex $u$ in $G$ such that $u \notin N_{\text {deg }}[v]$, then we say that the vertex $v$ is blind to the vertex $u$ with respect to degree-jump. Otherwise, we say that the vertex $v$ can see the vertex $u$.

Note that $K_{1}, P_{2}, P_{3}$ do not have blind vertices but the end vertices of a path graph $P_{n}, n \geq 4$, are blind to all internal vertices except two. Also, a vertex $v$ can always see itself because $v \in N_{\text {deg }}[v]$, which means that for a graph of order $n \geq 2$ a vertex can see at least two vertices. The property of a vertex seeing another vertex is not necessary commutative because it is possible that $u \notin N_{d e g}[v]$ and $v \in N_{d e g}[u]$. Then we have the following notion.

Definition 4. The peripheral number of a graph $G$, denoted by $\mathfrak{p}(G)$, is the number of vertices which can see all vertices of $G$.

Applications of the notion of blind vertices can be found in communication networks, social networks, monitoring systems, cryptology design and physical observation systems. Searching programs in space can be restricted by one-sided detection as well. Blindness may result from defined restrictions on communication range, distance or other meaningful graph theoretical properties. This new notion also relates to the concept of broadcasting in graphs.

Theorem 5. A connected graph $G$ has a vertex $v$ which can see all vertices of $G$ if and only if $d(v) \geq \max \{d(v, u): u \in V(G)\}$.

Proof. It is obvious that if $d(v) \geq \max \{d(v, u): u \in V(G)\}$, then the following two cases are to be considered.

(i) If $v$ is an end vertex of a diam-path in $G$, then for every vertex $u$ in $V(G), d(v, u) \leq d(v)$ implies $N_{d e g}[v]=V(G)$.

(ii) If $v$ is not an end vertex of any diam-path of $G$, then for every vertex $u$ in $V(G), d(v, u)<$ $d(v)$ implies $N_{\text {deg }}[v]=V(G)$.

From both cases, it follows that $v$ can see all vertices of $G$. If there exists a vertex $v$ that can see the vertex $u$, then it implies $d(v, u) \leq d(v)$ or, equivalently, $u \in N_{\text {deg }}[v]$. If $v$ can see all $u \in V(G)$, then $N_{\text {deg }}[v]=V(G)$. It means that $d(v) \geq \max \{d(v, u): u \in V(G)\}$.

The next proposition provides the peripheral number of certain cycle related graphs. Proofs are omitted because it can easily be verified by comparing $d(v)$ and $\max \{d(v, u): u \in V(G)\}$.

Proposition 2. (i) For a wheel graph $W_{1, n}$ we have $\mathfrak{p}\left(W_{1, n}\right)=n+1$.

(ii) For a double wheel graph $D W_{1, n}$ we have $\mathfrak{p}\left(D W_{1, n}\right)=2 n+1$.

(iii) For a helm graph $H_{n}$ we have $\mathfrak{p}\left(H_{n}\right)=n+1$.

(iv) For a closed helm graph $\mathrm{CH}_{n}$ we have $\chi_{r d j}\left(\mathrm{CH}_{n}\right)=2 n+1$. 
(v) For a prism graph $\Pi_{n}$ we have $\mathfrak{p}\left(\Pi_{n}\right)= \begin{cases}2 n, & 3 \leq n \leq 5, \\ 0, & \text { otherwise. }\end{cases}$

(vi) For a web graph $W_{n}$ we have $\mathfrak{p}\left(\Pi_{n}\right)= \begin{cases}2 n, & 3 \leq n \leq 5, \\ 0, & \text { otherwise. }\end{cases}$

(vii) For a flower graph $F_{n}$ we have $\mathfrak{p}\left(F_{n}\right)=2 n+1$.

(viii) For a djembe graph $D j_{n}$ we have $\mathfrak{p}\left(D j_{n}\right)=2 n+1$.

Theorem 6. For a graph $G$ we have $\mathfrak{p}(G) \leq \chi_{\text {rdj }}(G)$.

Proof. The result follows as a direct consequence of Theorem 5 because it is possible to have fewer vertices, each seeing all vertices of $G$. However, if all vertices can mutually see each other, then $\min \left\{\chi_{\text {rdj }}(G)\right\}=n=\mathfrak{p}(G)$.

\subsection{Sight matrix properties}

Let the vertices of a graph $G$ of order $n$ be labeled $v_{1}, v_{2}, v_{3}, \ldots, v_{n}$. Define the binary variable

$$
\text { (S) }\left(v_{i}\right)_{v_{j}}= \begin{cases}0, & \text { if } v_{i} \text { is blind to } v_{j} \\ 1, & \text { if } v_{i} \text { can see } v_{j} .\end{cases}
$$

For each vertex $v_{i}$ a sight vector defined by $\overrightarrow{\mathrm{S}\left(v_{i}\right)_{V(G)}}=\left(\mathrm{S}\left(v_{i}\right)_{v_{j}}: 1 \leq j \leq n\right)$ and a corresponding sight matrix defined by

$$
\mathrm{S}(G)=\left[\overrightarrow{\mathrm{S}\left(v_{i}\right)_{V(G)}}: 1 \leq j \leq n\right]=\left[\mathrm{S}\left(v_{i}\right)_{v_{j}}: 1 \leq i, j \leq n\right]
$$

exist.

Example 1. For a path graph $P_{5}$ and a cycle graph $C_{5}$ the respective sight matrices are

$$
S\left(P_{5}\right)=\left(\begin{array}{lllll}
1 & 1 & 0 & 0 & 0 \\
1 & 1 & 1 & 1 & 0 \\
1 & 1 & 1 & 1 & 1 \\
0 & 1 & 1 & 1 & 1 \\
0 & 0 & 0 & 1 & 1
\end{array}\right), \quad S\left(C_{5}\right)=\left(\begin{array}{lllll}
1 & 1 & 1 & 1 & 1 \\
1 & 1 & 1 & 1 & 1 \\
1 & 1 & 1 & 1 & 1 \\
1 & 1 & 1 & 1 & 1 \\
1 & 1 & 1 & 1 & 1
\end{array}\right) .
$$

Let the $n \times n$ identity matrix $I_{n}$ be as conventionally understood. Denote a matrix with complete 1-entries to be $I^{n}$. It follows easily that $S\left(C_{5}\right)=(S)$ for any super graph $H$ of order 5. It is easy to see that $S\left(K_{n}\right)=I^{n}$. Hence, it can be seen that a graph for which (S) $(G)=I^{n}$ is not unique. Furthermore, the diagonal entries of $S(G)$ are equal to 1 . But for the null graph $\mathfrak{N}_{n}$ of order $n$ we have $S\left(\mathfrak{N}_{n}\right)$ is equivalent to the identity matrix $I_{n}$.

Leading to the next result, we call $K_{1}$ a collapsed cycle and we call $K_{2}$ (or $P_{2}$ ) a flat cycle. We note that $K_{1}$ corresponds to a largest 0-regular connected graph with minimum edges for which $\chi_{r d j}(G)=1$. Similarly, $K_{2}$ corresponds to a largest 1-regular connected graph with minimum edges for which $\chi_{r d j}(G)=2$. A similar statement is true for $K_{5}$ as it corresponds to the largest 2-regular connected graph with minimum edges for which $\chi_{\text {rdj }}(G)=5$. 
An interesting question that arises in this context is: for $k \in \mathbb{N}_{0}$, to find a $k$-regular connected graph $G$ of largest order $n$ with minimum edges such that $\chi_{\text {rdj }}(G)=n$. Hence, (S) $(G)=I^{n}$ or in otherwords, $\mathfrak{p}(G)=n$. This family of $k$-regular graphs is called the Mphako graphs $^{1}$ and is denoted by $C_{n}^{+}(k)$.

\subsubsection{Mphako graphs}

The construction of a Mphako graph for a given $k \in \mathbb{N}, k \geq 2$, follows directly from the constructive proof of the next result.

Theorem 7. For $k \in \mathbb{N}, k \geq 2$, the corresponding Mphako graph $C_{n}^{+}(k)$ has order

$$
n=2 k^{2}-3 k+3 \text {. }
$$

Proof. Let $k \in \mathbb{N}, k \geq 2$. We begin with a vertex $v_{1}$ and extend consecutively along a path $v_{1} v_{2} v_{3} \cdots v_{2 k+1}$. Add the edge $v_{1} v_{2 k+1}$. From vertex $v_{2 k+1}$ extend along a further path $v_{2 k+1} v_{2 k+2} \ldots v_{4 k}$. Add the edge $v_{1} v_{4 k}$. Repeat this path extensions iteratively $(k-1)$ times. It is easy to verify that $n=2 k+(k-2)(2 k-1)+1=2 k^{2}-3 k+3$ is the maximum number of vertices that can be seen by $v_{1}$ with $d\left(v_{1}\right)=4$. With symmetry consideration add similar edges for vertices $v_{i}, 2 \leq i \leq n$. The graph resulting from this construction is the corresponding Mphako graph $C_{n}^{+}(k)$, since $d\left(v_{j}\right)=4,1 \leq j \leq n$, and $N_{d e g}\left[v_{j}\right]=V\left(C_{n}^{+}\right)(k)$ for all $i$ and a maximum.

It follows that $\varepsilon\left(C_{n}^{+}(k)\right)=\frac{1}{2} k\left(2 k^{2}-3 k+3\right)$. This number of edges is sharp.

Corollary 3. For $k \neq 3^{t}, t \in \mathbb{N}_{0}$, a Mphako graph $C_{n}^{+}(k)$ has odd number of vertices.

Proof. Let $t=3^{t}, t \in \mathbb{N}_{0}$. In the decimal number system, powers of 3 have the digits $1,3,9,7$ repeating cyclically in the $10^{0}$ column (unit's place) as $t$ increases through the non-negative integers. Hence, in

$$
\begin{aligned}
2 \cdot 3^{2 t}-3^{t+1}+3 & =2 \cdot 3^{2 t}-3\left(3^{t}-1\right)=2 \cdot 3^{2 t}-(2+1)\left(3^{t}-1\right) \\
& =2 \cdot 3^{2 t}-2\left(3^{t}-1\right)-\left(3^{t}-1\right)=\underbrace{2 \cdot 3^{2 t}}_{\text {even }}-2 \cdot \underbrace{\left(3^{t}-1\right)}_{\text {even }}-\underbrace{\left(3^{t}-1\right)}_{\text {even }}
\end{aligned}
$$

the expression equals an even number. By similar reasoning, $2 k^{2}-3 k+3$ is odd for $k \neq 3^{t}$, $t \in \mathbb{N}_{0}$.

It can be said that for any finite $n$ and $k \in\{i: 1 \leq i \leq n\}$, the Mphako graphs with even number of vertices are scarce. The reason for this scarcity is that for $t \in \mathbb{N}_{0}$, there are $t+1$ such Mphako graphs amongst the finite collection of Mphako graphs $\left\{C_{n}^{+}(k): 0 \leq k \leq t\right\}$. Hence, for $n \in \mathbb{N}_{0}$ let largest $t$ be such that $3^{t} \leq n$. Therefore, $t \leq \log _{3} n$. Randomly selecting a Mphako graph of even order from amongst the family $\left\{C_{n}^{+}(k): 0 \leq k \leq n\right\}$ has probability $\frac{1+\log _{3} n}{n}$. Since $\lim _{n \mapsto \infty}\left(\frac{1+\log _{3} n}{n}\right)=0$, the Mphako graphs of even order are said to be scarce.

Theorem 8. For a Mphako graph $C_{n}^{+}(k) k \geq 1$ we have

$$
\chi_{\text {deg }}\left(C_{n}^{+}(k)-e\right) \leq n-1=2 k^{2}-3 k+2,
$$

where $e \in E\left(C_{n}^{+}(k)\right.$.

\footnotetext{
${ }^{1}$ The second and third authors dedicate this family of graphs to the first author.
} 
Proof. We prove the result by mathematical induction. For $k=1$, the Mphako graph $C_{n}^{+}(1)=$ $K_{2}$. Hence, $n=2$ and clearly $\chi_{\text {deg }}\left(C_{2}^{+}(1)\right)=2>1=\chi_{\text {deg }}\left(K_{2}-e\right)$. For $k=2, C_{n}^{+}(2)=C_{5}$ and hence $n=5$ and clearly $\chi_{\text {deg }}\left(C_{5}^{+}(2)\right)=5>2=\chi_{\text {deg }}\left(C_{5}-e\right), e \in E\left(C_{5}\right)$.

Assume that the result holds for any $2 \leq k<\ell$ and let $k=\ell$. Without loss of generality, note that if any edge on the path $v_{1} v_{2} v_{3} \cdots v_{\ell+1}$ is deleted then vertex $v_{1}$ cannot see vertex $v_{\ell+1}$. Similarly, if any edge is deleted on the path $v_{1} v_{2 \ell+1} v_{2 \ell} \cdots v_{\ell+2}$ then vertex $v_{1}$ cannot see vertex $v_{\ell+2}$. Hence, the result follows by induction.

Note that for $k \geq 3$, the equality holds and thus $\chi_{\text {deg }}\left(C_{n}^{+}(k)-e\right)=n-1=2 k^{2}-3 k+2$, $e \in E\left(C_{n}^{+}(k)\right.$. It implies that for $k \in \mathbb{N}_{0}$, the Mphako graph $C_{n}^{+}(k)$ has maximum order and minimum size to ensure $\chi_{\text {deg }}\left(C_{n}^{+}(k)\right)=n$ and $S\left(C_{n}^{+}(k)\right)=I^{n}$.

Lemma 2. For $k \geq 2$, the Mphako graph $C_{n}^{+}(k)$ is $K_{n}$-free for $n \geq 3$.

Proof. It is easy to verify that a Mphako graph $k \geq 2$ is $K_{3}$-free. Therefore, it is $K_{n}$-free for $n \geq 3$.

Corollary 4. For $k \geq 2$, the Mphako graph $C_{n}^{+}(k)$ is 3-colorable.

Proof. From the constructive proof of Theorem 7 , it follows that $C_{n}^{+}(k), k \geq 2$, has induced odd cycles only. Therefore, $\chi\left(C_{n}^{+}(k)\right)=3$.

Corollary 5. For $k \geq 2$, diameter of the Mphako graph $C_{n}^{+}(k)$ is $k$.

Proof. For $k=2$, we know that $C_{n}^{+}(2)=C_{5}$ and $\operatorname{diam}\left(C_{5}\right)=2$. For $k=3$, the Mphako graph is a chorded cycle, namely, $C_{12}$ with chords $v_{i} v_{i+6}, 1 \leq i \leq 6$. Without loss of generality, $\operatorname{diam}\left(C_{n}^{+}(3)\right)=d\left(v_{1}, v_{5}\right)=3$ and is given by paths $v_{1} v_{7} v_{6} v_{5}$ or $v_{1} v_{12} v_{6} v_{5}$ or $v_{1} v_{12} v_{11} v_{5}$. By symmetry considerations similar diam-paths exist from all $v_{i}, 1 \leq i \leq 12$, to some vertex $v_{j}$. Hence, the result holds for $k=2,3$.

Assume that the result holds for $k=\ell$. For $k=\ell+1$, the path $v_{1} v_{2(\ell+1)+1} v_{2(\ell+1)} \ldots v_{\ell+1}$ is a diameter path (a path whose length is equal to the diameter of the graph under consideration). It follows easily that $\ell+1$ similar diameter paths exist from $v_{1}$ to $v_{\ell+1}$. By symmetry considerations, similar diameter paths of length $\ell+1$ exist from all $v_{i}, 1 \leq i \leq n=$ $2(\ell+1)^{2}-3(\ell+1)+3$, to some vertex $v_{j}$. Therefore, by mathematical induction, the result holds in general.

The Mphako graph is the solution to a degree diameter type problem. This particular problem has the specific condition that degree of all vertices equals $k$. Hence, the vertex degree is not bound to a maximum for some vertices as is the case in the classical degree diameter problem. Specifically $k$-regularity must hold. Recall the Moore bound for the classical degree diameter problem is given by $n_{d, k} \leq M_{d, k}$, where

$$
M_{d, k}= \begin{cases}2 k+1, & \text { if } d=2, \\ 1+d\left(\frac{(d-1)^{k}-1}{d-2}\right), & \text { if } d>2,\end{cases}
$$

and $n_{d, k}$ is the maximum number of vertices with degree at most $d$ and diameter $k$. With regards to the Mphako graphs the bound specialises to

$$
M_{k, k}= \begin{cases}2 k+1, & \text { if } k=2, \\ 1+k\left(\frac{(k-1)^{k}-1}{k-2}\right), & \text { if } k>2 .\end{cases}
$$

For $k=2$, the graph $C_{n}^{+}(2)=C_{5}$, the order equals the upperbound. However, for $k>2$ we have the next result. 
Proposition 3. For $k>2$, an upper bound for the order of the Mphako graph $C_{n}^{+}(k)$ is given by $v\left(C_{n}^{+}(k)<M_{k, k}\right.$.

Proof. Consider the real valued inequality $2 x^{2}-3 x+3 \geq 1+x\left(\frac{(x-1)^{k}-1}{x-2}\right)$, where $x \in \mathbb{R}, k \in \mathbb{N}$, $k>2$. Therefore,

$$
\begin{gathered}
(x-2)\left(2 x^{2}-3 x+2\right) \geq x(x-1)^{k}-x \\
2 x^{3}-7 x^{2}+9 x-4 \geq x(x-1)^{k}=x^{k+1} \pm a_{1} x^{k} \pm a_{2} x^{k-1} \pm \ldots \pm a_{k-1} x .
\end{gathered}
$$

Inequaliy (1) presents a contradiction because the leftside is a polynomial of order 3 while the rightside is a polynomial of order $k+1$ and the unique mutual real root is at $(1,0)$. Hence, $2 x^{2}-3 x+3<1+x\left(\frac{(x-1)^{k}-1}{x-2}\right), k>2$. Therefore, for the discrete case $x=k$, it follows that $v\left(C_{n}^{+}(k)<M_{k, k}\right.$.

\section{Conclusion}

The paper served as an introduction to the new notion of the rainbow degree-jump coloring of a graph. The rainbow degree-jump coloring of a prism graph suggest that researching the Cartesian product of graphs with respect to rainbow degree-jump coloring could be worthy. Similarly, the study of rainbow degree-jump coloring for the other known graph products remains open. Other graph operations such as the corona of two graphs, the line graph, the complement graph and others offer scope for further research.

The authors view the introduction of the new family of Mphako graphs as interesting cycle related graphs which is open for further research in various graph theoretical domains.

Problem 1. For $k \geq 2$ and for $2 k^{2}-3 k+3<m<2(k+1)^{2}-3(k+1)+3$ find the minimum number of edges in a graph $G$ such that $\chi_{\text {deg }}(G)=m$.

Problem 2. For two graphs $G, H$ with a diameter path $v_{i}$ to $v_{j}$ and $u_{l}$ to $u_{k}$, in each respectively, the string graph denoted by $G \rightsquigarrow H$ is obtained by adding the edge $v_{j} u_{l}$ or $v_{j} u_{k}$. To string from $G \rightsquigarrow H$ to graph $M$ which has a diameter path $w_{s}$ to $w_{t}$, to obtain $(G \rightsquigarrow H) \rightsquigarrow M$, add the edge $u_{k} w_{s}$ or $u_{k} w_{t}$. If each graph graph $G_{i}, 1 \leq i \leq t$, permits a rainbow degree-jump coloring, find the combination that results in a string graph $G$ such that

$$
\begin{aligned}
\chi_{r d j}(G)=\max \left\{\chi_{r d j}\left(\left(\left(\cdots\left(G_{i} \rightsquigarrow G_{i+1}\right) \rightsquigarrow \cdots\right) \rightsquigarrow G_{t-1}\right) \rightsquigarrow G_{t}\right)\right): \\
\\
\quad \text { over all combinations of the numbers } 1 \leq i \leq t\} .
\end{aligned}
$$

Problem 3. The notion of blind vertices has been introduced. It suggests the notion of a degreejump domination set of $G$. That is a minimum subset $X \subseteq V(G)$ such that $\bigcup_{v \in X} N_{\text {deg }}[v]=V(G)$. The cardinality of $X$ is called the degree-jump domination number of $G$ and is denoted by $\gamma_{d j}(G)$. This notion offers a new direction of research. 


\section{References}

[1] Bondy J.A., Murty U.S.R. Graph theory. Springer, New York, 2008.

[2] Sudev N.K., Chithra K.P., Shiny E.A. On equitable coloring parameters of certain wheel related graphs. Contemp. Stud. Discrete Math. 2017, 1 (1), 3-12.

[3] Harary F. Graph theory. Narosa Publ. House, New Delhi, 2001.

[4] Kok J., Naduvath S., Jamil M.K. Rainbow neighbourhood number of graphs. Proyecciones J. Math. 2019, 38 (3), 469-484. doi:10.22199/issn.0717-6279-2019-03-0030

[5] Kok J., Naduvath S., Mary U., Seles M.J. On chromatic core subgraph of simple graphs. Contemp. Stud. Discrete Math. 2018, 2 (1), 13-20.

[6] Kok J., Naduvath S., Susanth C., Kalayathankal S.J. A note on the rainbow neighbourhood number of graphs. Nat. Acad. Sci. Lett. 2019,42 (2), 135-138.

[7] Kok J., Naduvath S., Susanth C., Kalayathankal S.J. Some new results on the rainbow neighbourhood number of graphs. Nat. Acad. Sci. Lett. 2019, 42 (3), 249-252.

[8] Susanth C., Sudev N. K., Kalayathankal S.J. On chromatic curling number of graphs. Contemp. Stud. Discrete Math. 2017, 1 (1), 21-30.

[9] West D.B. Introduction to graph theory. Pearson Education Inc., Delhi, 2001.

Received 07.07.2020

Revised 13.02.2021

Мфако-Банда Е.Г., Кок Аж., Надуват С. Веселкове степенево-стрибкове розфарбування графів // Карпатські матем. публ. - 2021. - Т.13, №1. - С. 229-239.

У цій статті ми вводимо нове поняття веселкового степенево-стрибкового розфарбування графа. $\Delta$ я вершини $v \in V(G)$ нехай степенево-стрибковий замкнений окіл $v$ буде визначений як $N_{\text {deg }}[v]=\{u: d(v, u) \leq d(v)\}$. Належне розфарбування графа $G$ буде називатись веселковим степенево-стрибковим розфарбуванням $G$, якщо для всіх $v$ з $V(G), c\left(N_{d e g}[v]\right)$ містить принаймні по одному з кожного класу кольорів. Ми визначили необхідну і достатню умову того, що граф $G$ допускає веселкове степенево-стрибкове розфарбування. Також, ми визначили веселкове степенево-стрибкове хроматичне число, яке позначаємо $\chi_{r d j}(G)$, Аля деяких класів циклічно відносних графів.

Ключові слова і фрази: веселкове степенево-стрибкове розфарбування, веселкове степеневострибкове хроматичне число, невидима вершина, граф Мфако, межа Мура. 